\title{
Fibrolipomatous hamartoma of the median nerve
}

\begin{abstract}
Authors:
Leon Malan ${ }^{1}$

Abraham F. Bezuidenhout ${ }^{1}$

Ebrahim Banderker ${ }^{1}$

\section{Affiliations:}

${ }^{1}$ Department of Radiology,

Stellenbosch University,

South Africa

Correspondence to:

Leon Malan

Email:

Inmalan86@gmail.com

\section{Postal address:}

Ravensteyn Road, Camps

Bay, Cape Town 8005,

South Africa

Dates:

Received: 04 June 2015

Accepted: 11 Aug. 2015

Published: 11 Dec. 2015

How to cite this article: Malan L, Bezuidenhout $A F$, Banderker, E.

Fibrolipomatous hamartoma of the median nerve. $\mathrm{S}$ Afr J Rad. 2015;19(2); Art. \#886, 3 pages. http://dx.doi. org/10.4102/sajr.v19i2.886

\section{Copyright:}

(C) 2015. The Authors. Licensee: AOSIS

OpenJournals. This work is licensed under the Creative Commons Attribution License.
\end{abstract}

Read online:
Fibrolipomatous hamartoma is a rare benign tumour most commonly affecting the median nerve and is characterised by fibro-fatty nerve infiltration. It results in fusiform nerve enlargement with a pathognomonic 'spaghetti-like' imaging appearance. Patients present with numbness and paraesthesia and later with motor deficits in the affected nerve distribution. The condition frequently coexists with macrodystrophia lipomatosa in up to two-thirds of cases.

\section{Introduction}

Fibrolipomatous hamartoma, neural fibrolipoma or lipomatosis of the median nerve as described by the WHO is a rare benign tumour. ${ }^{1}$ Fibrolipomatous hamartoma is characterised by mature adipocyte proliferation within the nerve separating axonal bundles within the perineurium. Perineural and endoneural fibrosis thicken the axonal bundles, which are interspersed throughout the proliferative fat. ${ }^{2}$ The most commonly affected nerve is the median nerve $(66 \%-80 \%)$, followed by the radial nerve, the ulnar nerve, the dorsal nerves of the foot, the brachial plexus and the cranial nerves. ${ }^{2,3,4}$ The diagnosis is made primarily with radiological imaging. ${ }^{5}$

\section{Case presentation}

A five-year-old healthy boy presented with a one-year history of a slowly growing mass over the volar aspect of the left wrist with increasing paraesthesia and a decreasing range of movement.

Conventional radiography demonstrated mild soft tissue swelling over the volar aspect of the wrist with no underlying bony abnormality (Figure 1).

Ultrasound evaluation of the soft tissue mass demonstrated fusiform enlargement of the median nerve with alternating hypoechoic and hyperechoic linear bands, resulting in a 'spaghetti-like' appearance (Figure 2) on longitudinal images and a 'coaxial cable-like' appearance (Figure 3) on transverse images.

MRI investigation followed which also showed fusiform enlargement of the median nerve fascicles with fibro-fatty proliferation in a characteristic 'spaghetti-like' (Figure 4) and 'coaxial cable-like' (Figure 5) appearance of the median nerve. The enlarged median nerve resulted in crowding of the carpal tunnel and compression of the adjacent flexor tendons. There was no associated macrodactyly.

MR findings were pathognomonic of a fibrolipomatous hamartoma of the left median nerve.

\section{Discussion}

Clinically, patients present with a gradually enlarging, non-tender palpable mass. ${ }^{2}$ Neurological symptoms are often present in the distribution of the specific nerve. If the median nerve is involved, these symptoms may overlap with the symptoms of carpal tunnel syndrome. ${ }^{6}$ Neurological symptoms include numbness and paraesthesia with motor deficits occurring late. ${ }^{3}$ Nerve conduction studies demonstrate no stimulation response of the affected nerve. The underlying aetiology is unknown; however, some authors believe the proliferation is secondary to nerve irritation, inflammation or trauma. ${ }^{5}$

Fibrolipomatosis hamartoma was first described in 1953. Johnson and Bonfiglio coined the term 'fibrolipomatous hamartoma' as they differentiated it from macrodystrophia fibromatosa and neurofibroma in neurofibromatosis. ${ }^{7}$

MRI is now considered the gold standard in imaging fibrolipomatous hamartoma and is considered to be definitive if the typical imaging findings are present, obviating the need for 


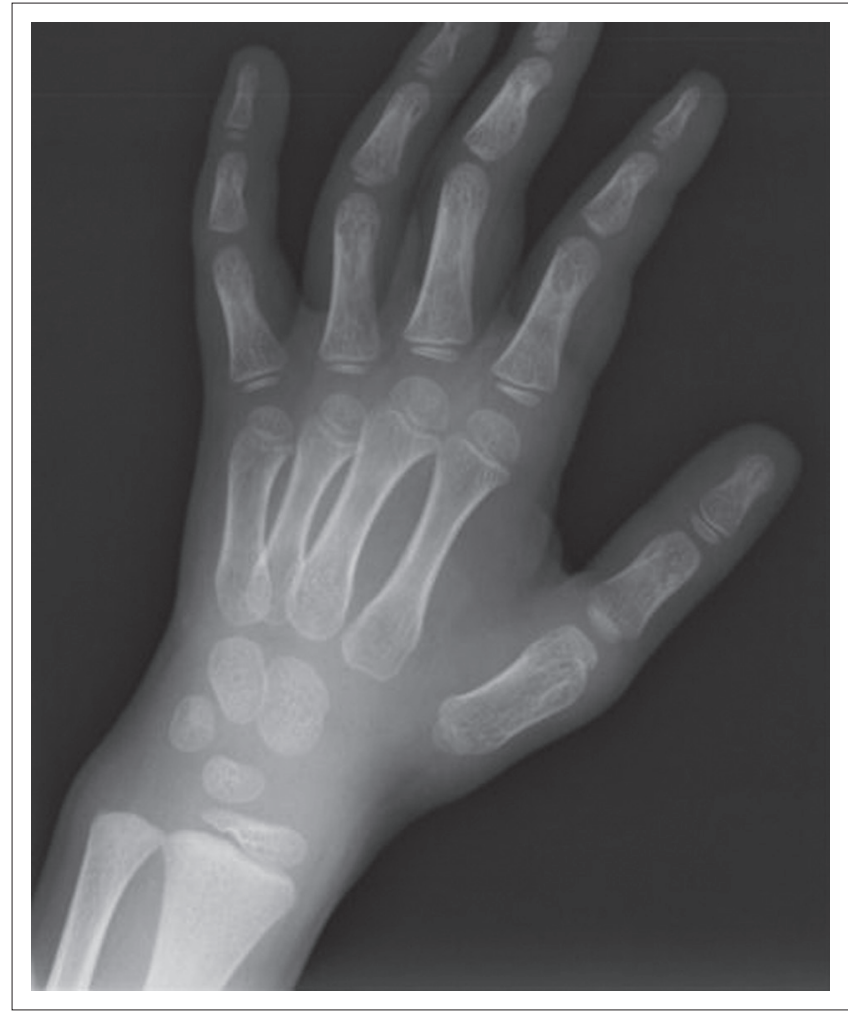

FIGURE 1: Conventional radiography demonstrates mild soft-tissue swelling over the volar aspect of the wrist with no underlying bony abnormality.

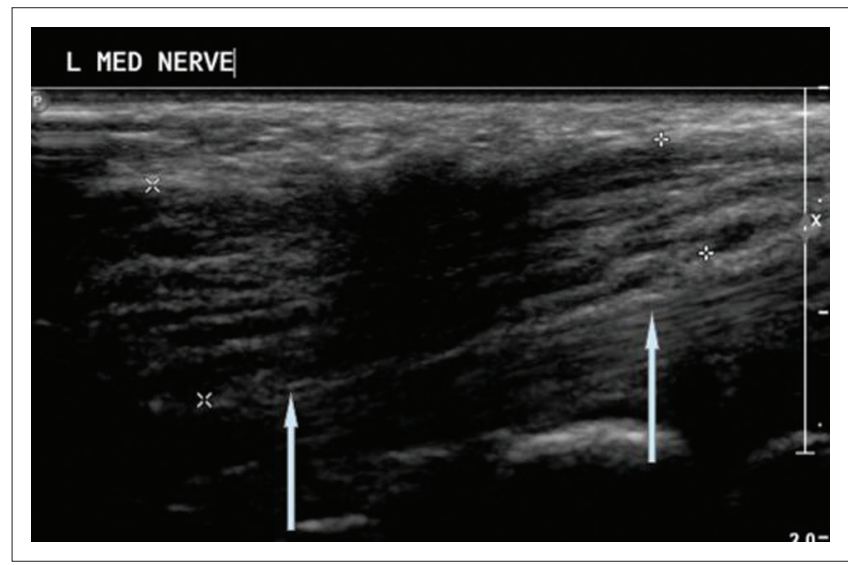

FIGURE 2: Longitudinal ultrasound image demonstrates fusiform enlargement of the median nerve with alternating hypoechoic and hyperechoic linear bands, resulting in a 'spaghetti-like' appearance (white arrows).

further investigation. ${ }^{5}$ MRI demonstrates a high signal intensity fusiform mass on $\mathrm{T} 1$ and $\mathrm{T} 2$ weighted imaging representing the adipose proliferation. Signal void is appreciated on fat suppression techniques. The mass has a characteristic 'coaxial cable-like' appearance on axial images and a 'spaghetti-like' appearance on sagittal images which represent the circumferentially fibrosed neural bundles within the proliferative fat. No contrast enhancement is present after gadolinium administration. ${ }^{3,8,4}$

Ultrasonographic imaging of fibrolipomatous hamartoma also demonstrates the characteristic hypoechoic 'coaxial cable-like' mass encased by an echogenic substrate, and some authors believe such imaging to be as effective as MRI

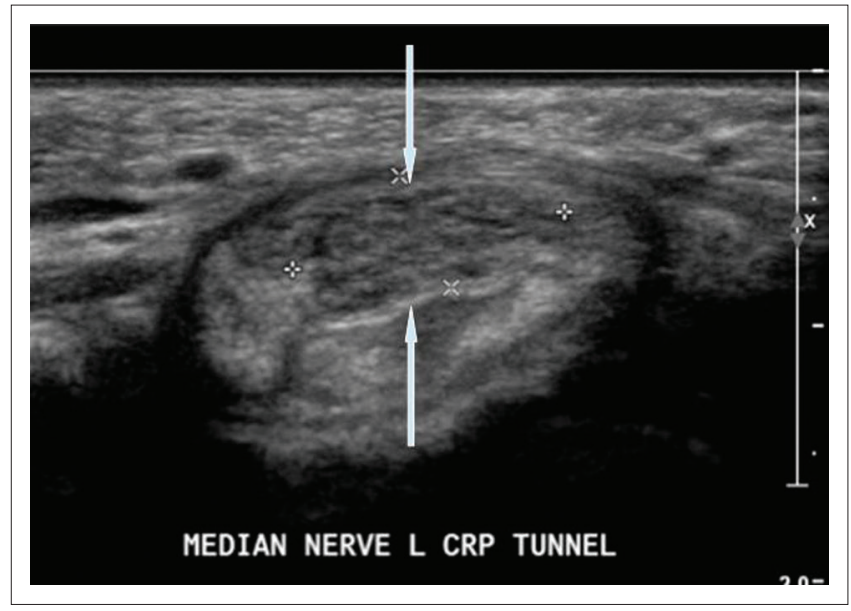

FIGURE 3: Transverse ultrasound image demonstrates enlargement of the median nerve with alternating hypoechoic and hyperechoic dots resulting in a 'coaxial cable-like' appearance (white arrows).

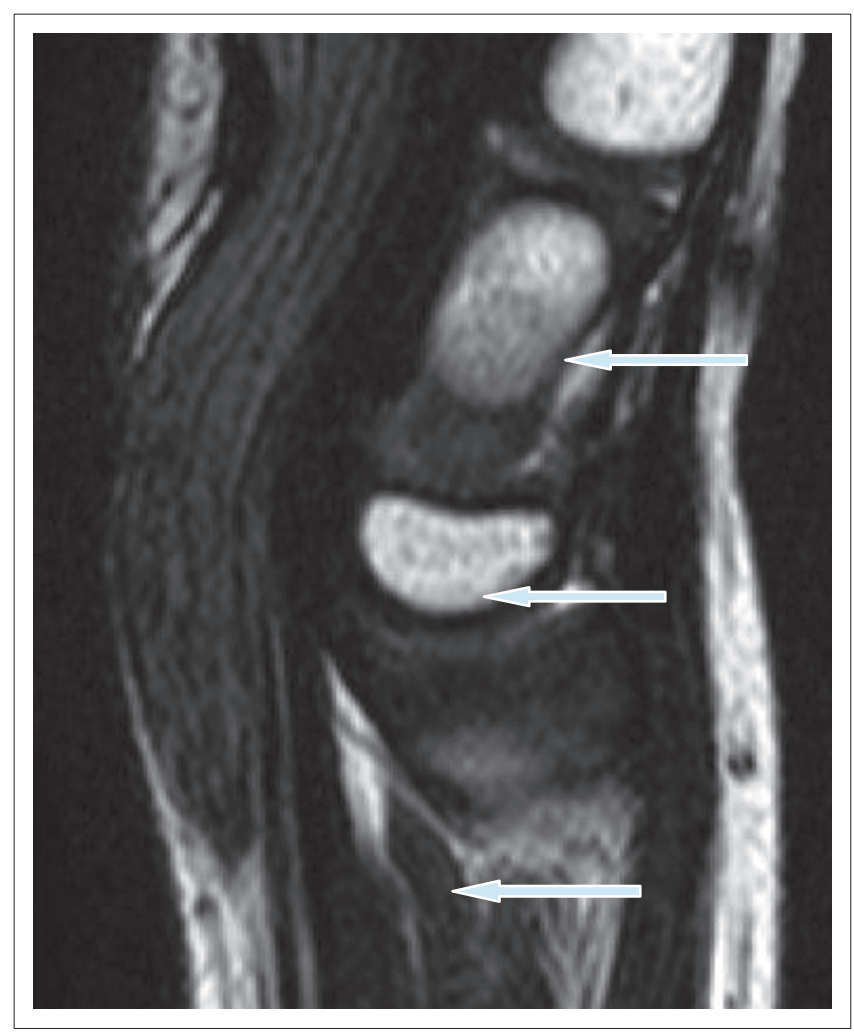

FIGURE 4: T1WI sagittal MR image demonstrates fusiform enlargement of the slightly hyperintense median nerve fascicles with a characteristic 'spaghetti-like' appearance (white arrows).

in establishing the diagnosis; however, no comparative study is available. ${ }^{9}$

Macrodystrophia lipomatosis is associated with fibrolipomatous hamartoma in $22 \%-67 \%$ of cases. ${ }^{3,7}$ It is a non-hereditary congenital macrodactyly and is characterised by diffuse proliferation of all the tissues involved: skin, bone and fatty proliferation between the nerve fascicles. This association has a 2:1 female predominance but the relationship between the two entities is unknown. ${ }^{5}$

In 1994, Guthikonda et al. further divided lipid-containing masses according to their relationship with the parent nerve. 


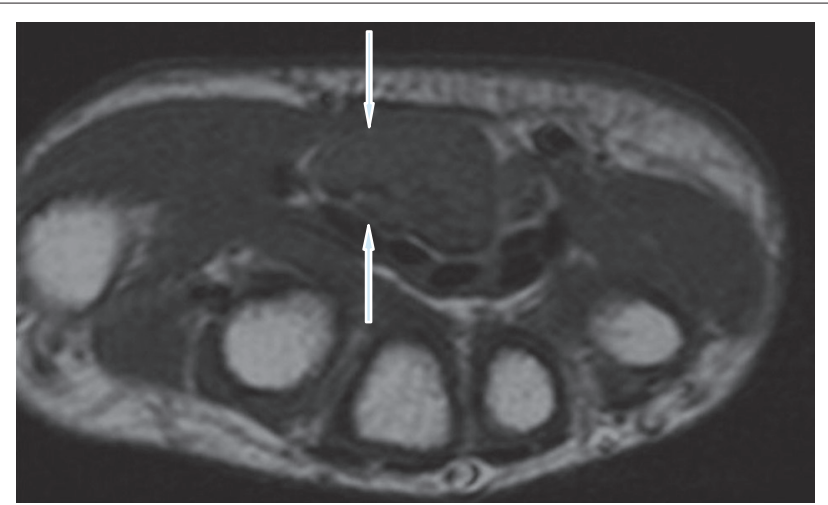

FIGURE 5: T1WI axial MR image demonstrates enlargement of the slightly hyperintense median nerve fascicles with a characteristic 'coaxial cable-like' appearance (white arrows)

This characterisation included: soft tissue lipoma, intraneural lipoma, macrodystrophia lipomatosis and fibrolipomatous hamartoma. Soft tissue lipomas are differentiated by their position outside the nerve and a lack of any neural proliferation. Similarly, intraneural lipomas demonstrate focal lipid masses separated from individual nerve bundles and in the absence of neural proliferation. ${ }^{10}$

Currently there is no effective treatment for fibrolipomatous hamartomas and conservative management is preferred if symptoms are minimal. Surgical resection risks permanent nerve damage and minor surgical decompression procedures, that is, carpal tunnel release, for symptomatic relief are more commonly performed. .,11 $^{11}$

\section{Conclusion}

Fibrolipomatous hamartoma is a benign fibrofatty infiltration of the median nerve with associated fibrosis of the neural bundles. It can be diagnosed on MRI findings alone due its typical 'co-axial cable-like' and 'spaghetti-like' appearances on longitudinal and axial imaging respectively. These features were well demonstrated in our case and thus made the diagnosis straightforward. It is important always to consider macrodystrophia lipomatosis due to its strong association with fibrolipomatous hamartoma and to differentiate these from other lipid-containing soft tissue masses.

\section{Acknowledgements Competing interests}

The author declares that they have no financial or personal relationships which may have inappropriately influenced them in writing this article.

\section{References}

1. Nielsen GP. Lipomatosis of nerve. In: Fletcher CDM, Unni KK, Mertens F, editors. Pathology and genetics of tumours of soft tissue and bone. Lyon: IARC Press, 2002; p. 24-25.

2. Nouira $\mathrm{K}$, Belhiba $\mathrm{H}$, Baccar $\mathrm{S}$, et al. Fibrolipoma of the median nerve. Joint Bone Spine. 2007;74:98-99. PMID: 17178460, http://dx.doi.org/10.1016/j. jbspin.2006.02.012

3. Van Breuseghem I, Sciot R, Pans S, Geusens E, Brys P, De Wever I. Fibrolipomatous hamartoma in the foot: Atypical MR imaging findings. Skeletal Radiol. 2003;32:651655. PMID: 12955352, http://dx.doi.org/10.1007/s00256-003-0684-3

4. Marom EM, Helms CA. Fibrolipomatous hamartoma: Pathognomonic on MR imaging. Skeletal Radiol. 1999;28:260-264. PMID: 10424331, http://dx.doi. org/10.1007/s002560050512

5. Tahiri Y, Xu L, Kanevsky J, Luc M. Lipofibromatous hamartoma of the median nerve: A comprehensive review and systematic approach to evaluation, diagnosis, and treatment. J Hand Surg Am. 2013;38(10):2055-2067. PMID: 23684521, http://dx.doi.org/10.1016/j.jhsa.2013.03.022

6. Johnson RJ, Bonfigilio M. Lipofibromatous hamartoma of the median nerve. J Bone and Joint Surg. 1969;51:984-990.

7. Silverman TA, Enzinger FM. Fibrolipomatous hamartoma of nerve. A clinicopathologic analysis of 26 cases. Am J Surg Pathol. 1985;9:7-14. PMID: 3970300.

8. Campbell CS, Wulf RF. Lipoma producing a lesion of the deep branch of the radial nerve; case report. J Neurosurg. 1954;11:310-311. PMID: 13163731.

9. Toms AP, Anastakis D, Bleakney RR, Marshall TJ. Lipofibromatous hamartoma of the upper extremity: A review of the radiologic findings for 15 patients. Am J Roentgenol. 2006;186:805-811. PMID: 16498111, http://dx.doi.org/10.2214/ AJR.04.1717

10. Guthikonda M, Rengachary SS, Balko MG, van Loveren H. Lipofibromatous hamartoma of the median nerve: Case report with magnetic resonance imaging correlation. Neurosurgery. 1994;35(1):127-132. PMID: 7936133, http://dx.doi. org/10.1227/00006123-199407000-00019

11. Paletta FX, Senay LC Jr. Lipofibromatous hamartoma of median nerve and ulnar nerve: Surgical treatment. Plast Reconstr Surg. 1981;68(6):915-921. PMID: 7301986 . 